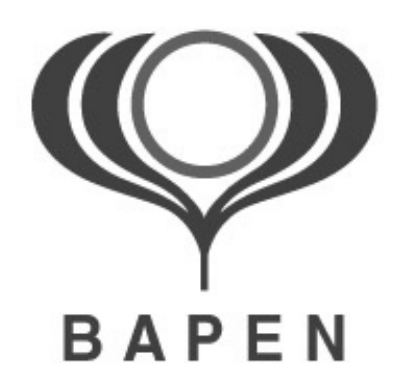

Advancing Clinical Nutrition

Reg. Charity No: 1023927

\title{
THE 'MUST’ REPORT
}

\section{Nutritional screening of adults: a multidisciplinary responsibility}

\section{Executive \\ Summary}

Professor Marinos Elia

Chairman of MAG and Editor

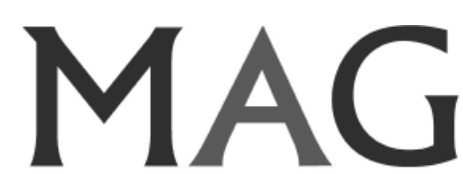

Malnutrition Advisory Group

A Standing Committee of BAPEN 


\section{Executive summary}

This report examines the need to screen for malnutrition in clinical practice, sets out the criteria that need to be fulfilled, and describes the development and use of the 'Malnutrition Universal Screening Tool' ('MUST') for adults, which takes these criteria into account. The tool primarily aims to identify risk of poor protein-energy status, rather than status of individual nutrients. It is linked to a care plan, which can vary according to healthcare setting, local policies, and resources. Guidance on undertaking measurements using 'MUST' is provided.

\section{Section A: Screening for malnutrition: a multidisciplinary responsibility}

\section{Malnutrition (undernutrition) and overweight/obesity as major clinical and public health problems in the UK}

1.1 Malnutrition (undernutrition): Underweight (BMI $<20 \mathrm{~kg} / \mathrm{m}^{2}$ ) is typically present in $10-40 \%$ of patients admitted to hospital, but malnutrition risk, established using the 'MUST', is even greater. In the general population, it is estimated that one in seven subjects aged 65 years and over has a medium or high risk of malnutrition, but the prevalence is higher in subjects who are institutionalised than those who are free living (i.e. living in their own homes). Malnutrition predisposes to disease, delays recovery from illness, and adversely affects body function, well-being and clinical outcome. There is no formal economic evaluation of disease-related malnutrition but it is estimated that the cost is greater than that of obesity.

1.2 Obesity: The incidence of obesity (body mass index (BMI) $>30 \mathrm{~kg} / \mathrm{m}^{2}$ ) is increasing in both adults and children, and currently affects one in five adults. It predisposes to many health problems, including heart disease, diabetes, high blood pressure and osteoarthritis, with an estimated annual cost to the economy of over $£ 2$ billion, of which $£ 0.5$ billion represents a direct cost to the National Health Service.

\section{Malnutrition (undernutrition): under-recognised and undertreated}

Malnutrition is often unrecognised and untreated in hospitals (both in-patients and out-patients), nursing homes and in the community, causing concern among a wide range of health professionals, national organisations and colleges, UK government departments, and the Council of Europe. Despite this, there are no national guidelines for commissioners and planners of healthcare.

\section{Inadequate nutritional care}

Nutritional care is frequently inadequate because of diffuseness of responsibility, lack of an integrated infrastructure for dealing with nutritional problems within and between different healthcare settings, poor education, and lack of consistent criteria to identify and treat malnutrition. There are well over 50 published nutrition screening tools and many more unpublished tools in clinical use, taking anything from two minutes to over thirthy minutes to complete. These differ in the criteria they use, the weighting factors applied to the criteria, the scoring systems, the intended users (who are sometimes not specified), and the tool's practical acceptability in routine clinical practice. Many have not been tested for reliability or validity, and many lack an evidence base. Furthermore, several different tools may be in use in the same hospital and in the community, contributing to confusion about how to recognise and manage malnutrition. 


\section{Common principles of nutritional screening and care}

The problems and principles of nutritional screening are illustrated by examining the common threads that apply to underweight and overweight children and adults, including pregnant and lactating women. The section on children is also included because nutritional problems in children become nutritional problems in adults, especially if there is inadequate continuity of care.

\section{Nutritional screening and assessment}

Nutritional screening, which is the focus of this report, refers to a rapid, general, often initial evaluation undertaken by nurses, medical or other staff, to detect significant risk of malnutrition and to implement a clear plan of action, such as simple dietary measures or referral for expert advice. Nutritional assessment is a more detailed, more specific, and more in-depth evaluation of nutritional status by an expert, so that specific dietary plans can be implemented, often for more complicated nutritional problems. This difference is often misunderstood, contributing to confusion.

\section{Recommendations}

6.1 Routine use of a nutritional screening tool: A nutritional screening tool should be used routinely for patients admitted to hospitals and care homes. It should also be used with new patients attending general practitioner (GP) surgeries, in those aged 75 years and over undertaking routine annual health assessments, in vulnerable groups, and in those for whom there is clinical concern (e.g. those who are frail and elderly, the poor and socially isolated, and those with severe diseases and disabilities). Screening should be repeated at intervals depending on the healthcare setting and clinical condition. The same tool should be used to screen patients at risk of malnutrition as they move from one healthcare setting to another.

6.2 Characteristics of the nutrition screening tool: (i) The screening tool should be: practical (easy to understand, easy and quick to complete, and acceptable to patients/subjects and healthcare workers), reliable, valid and evidence based. It should also incorporate a scoring system that is applicable and relevant to different clinical conditions and care settings, and be linked to a care plan. (ii) The screening tool should address the following: current weight status (e.g. underweight or obesity using BMI), as well as recent past and likely future change in weight, both of which are related to food intake and disease severity. Objective measures should be used whenever possible, and less objective measures when necessary. (iii) The screening tool should aid rather than replace clinical judgment.

6.3 The nutritional screening programme: After application of the screening test, which aims to identify patients at risk of malnutrition, it is often necessary to undertake more detailed and more specific assessment (e.g. by referral to a dietitian or nutritional support team) as part of a care plan. The policy for the entire screening programme - from the initial test to assessment, treatment, monitoring, documentation, communication and evaluation - should be established by a multidisciplinary group of healthcare workers, according to recommended procedures for screening and guideline development, and according to local resources.

6.4 Weighing scales and stadiometers: Accurate and reliable weighing scales and stadiometers, for measuring weight and height respectively, should be available to all hospital wards, outpatient clinics, care homes, GP surgeries, and other healthcare settings. 


\subsection{Consistent framework and principles for nutritional screening programmes:}

Screening programmes for malnutrition in children and obesity in adults and children should follow the same principles as screening for malnutrition in adults. Unintentional weight loss in obese individuals should be taken seriously since it may suggest the presence of an underlying disease. In contrast, persistent weight gain in children may be inadequate to sustain normal growth. Adult malnutrition screening programmes should note obesity when it exists, link with childhood nutritional programmes, and cater for individuals in different healthcare settings using the same sound principles and procedures operating through an appropriate infrastructure. Screening tests and programmes should be evaluated with respect to their application and effectiveness.

6.6 Infrastructure and clinical governance: Commissioners, planners and providers of healthcare should be part of a coherent and integrated infrastructure, extending through all levels of the health and care service from Government departments, regional and local services, to individual health and care workers. This continuum should foster the development of nutrition strategies and the establishment of responsibilities and policies for the prevention and treatment of malnutrition across conditions and healthcare settings. The effectiveness of such policies, including nutritional screening programmes, education, training and personal development plans, should be monitored and evaluated.

\section{Section B: Validity, reliability and practicality of using the 'Malnutrition Universal Screening Tool' ('MUST')}

\section{The acronym 'MUST'}

Although it is recognised that the 'Malnutrition Universal Screening Tool' for adults may not effectively screen for deficiencies or toxicities of certain micronutrients, it can be readily applied to all types of patient groups in different healthcare settings. These include those with eating disorders, mental health problems and critical illness, as well as those with fluid disturbances, pregnancy, or lactation. It uses the same conceptual framework for all adults, employing more subjective criteria (e.g. when there are fluid disturbances) or modified criteria (e.g. weight changes during pregnancy) in some circumstances. The acronym, which is presented in inverted commas to indicate these caveats, is also used as a means of encouraging screening for malnutrition in a range of care settings where this is currently not carried out routinely.

\section{Development of the evidence base}

'MUST' was developed for use in adults in response to the criteria set out in section A of this report. It provides a theoretical and practical framework for the clinical detection and management of nutritionally responsive conditions, caused by physical and psychosocial problems. The tool is simple, valid, and reliable, and is suitable for practical use by a range of healthcare workers operating in different healthcare settings.

\section{The tool and its components}

'MUST' was developed by a multi-disciplinary group of health professionals and patients to detect both undernutrition (poor protein-energy status, referred to as malnutrition in this document) and obesity in adults of different ages and diagnoses in different healthcare settings. The tool involves assessment of weight status (BMI), 
change in weight, and the presence of an acute disease resulting in no dietary intake for more than 5 days (or likely to result in no dietary intake for more than 5 days). It can also be viewed as tracing the clinical journey of the patient, from the past (history of unintentional weight change) to the present (current weight status or $\mathrm{BMI}$ ) and into the future (likely effect of underlying condition). All three components can independently influence clinical outcome. In situations where weight and height cannot be measured, self-reported measurements, other surrogate measurements, and clinical judgment can be used to reliably estimate underweight, obesity and overall malnutrition risk. The tool categorises subjects into low, medium, or high risk of malnutrition and identifies the obese. It provides guidance on the interpretation of measurements, and suggests appropriate care plans, which can be modified to take into account local policy and resources.

\section{Validity}

The tool has face validity, content validity, concurrent validity with a range of other screening tools, and predictive validity. In hospitals (medical, elderly and orthopaedic wards), 'MUST' predicts length of stay (e.g. up to 2-4 times longer in high than low risk patients in elderly medical wards), discharge destination (e.g. to nursing homes and other hospitals from orthopaedic wards), and mortality after controlling for age. In the community, 'MUST' predicts rates of hospital admissions and GP visits, and shows that appropriate nutritional intervention improves outcome.

\section{Reliability and internal consistency}

The tool is internally consistent and reliable. It has very good to excellent reproducibility when different observers assess the same patients in hospitals (in-patients and out-patients), GP surgeries, and care homes (kappa values between 0.8 and 1.0).

\section{Practicality}

The tool has been found to be easy and quick to use and acceptable to both patients/subjects and healthcare workers.

\section{Further evidence based consideration}

Justification is provided for the use of an acute disease effect in 'MUST', equal weightings of the three component categories of 'MUST' (BMI, weight loss and acute disease effect), and the lower boundary BMI of $20 \mathrm{~kg} / \mathrm{m}^{2}$ for the elderly.

\section{Section C: Guidance on undertaking measurements and using 'MUST'}

\section{Measurements}

Procedures for measuring weight, height, and establishing BMI and weight loss are described, together with methods for estimating them (from ulna length, knee height, demispan, mid-upper arm circumference (MUAC)) when they cannot be measured directly.

\section{Interpretation and use of the tool}

Guidance is provided on how to use the tool in a range of situations, particularly those in which confounding factors influence the interpretation of weight change and BMI. Considerations and alternative measures relevant to these situations are 
summarised below.

15.1 Fluid disturbances: (i) BMI A low BMI is more significant if underweight is present with than without oedema. In the presence of barely detectable oedema, a correction can be applied by subtracting $2-3 \mathrm{~kg}$ from the measured weight. MUAC can also be used as an indicator of underweight when there is oedema or excess fluid in the legs or trunk (including ascites) but not in the arms. Alternative strategies are to re-measure weight after correcting disturbances in hydration status, and to classify subjects as thin, acceptable weight, or overweight by inspecting them, noting if they are obviously wasted (very thin) or very overweight (obese). (ii) Weight change When there are large and fluctuating fluid shifts, a history of changes in appetite and presence of conditions likely to lead to weight change can be used as part of an overall subjective evaluation of malnutrition risk, which categorises patients into low or medium/high risk categories.

15.2 Lactation: (i) BMI Use measured BMI. (ii) Weight change As for oedema. 15.3 Pregnancy: (i) Pre-pregnancy BMI Measurements of weight and height before pregnancy (or during early pregnancy, which is associated with little change in body weight) or recalled values can be used to estimate pre-pregnancy BMI; MUAC changes little during pregnancy and can be used to establish approximate pre-pregnancy BMI categories. (ii) Weight change Weight gains $<1 \mathrm{~kg}(<0.5 \mathrm{~kg}$ in the obese) or $>3 \mathrm{~kg}$ per month during the $2 \mathrm{nd}$ and 3 rd trimester generally require further evaluation.

15.4. Critical illness: Acute disease effect (no or unlikely dietary intake for $>5$ days) Most patients in typical intensive care units are at risk of malnutrition.

15.5 Plaster casts: Synthetic and Plaster of Paris casts for upper limbs weigh $<1 \mathrm{~kg}$; those for the lower leg and back weigh $0.9-4.5 \mathrm{~kg}$ depending on the material and site (see section C.3.2.6).

15.6 Amputations: Weight adjustments can be made from knowledge of the weight of missing limb segments: upper limb $4.9 \%$ of body weight (upper arm $2.7 \%$; forearm 1.6\%; hand 0.6\%); lower limb 15.6\% (thigh 9.7\%; lower leg 4.5\%; foot $1.4 \%$ ).

\section{The overall risk of malnutrition}

This is linked to a care plan, but the operational pathways can vary from centre to centre to take into account specific groups of patients and the available resources. 